\begin{tabular}{|l|c|c|c|c|} 
Revista Praxis & ISSN: $1657-4915$ & Vol. 12 & $63-77$ & Enero - Diciembre de 2016 \\
\hline
\end{tabular}

\title{
OBJETOS VIRTUALES DE APRENDIZAJE Y EL DESARROLLO DE APRENDIZAJE AUTÓNOMO EN EL ÁREA DE INGLÉS
}

\author{
VIRTUAL LEARNING OBJECTS AND THE DEVELOPMENT OF AUTONOMOUS LEARNING \\ IN ENGLISH
}

\author{
Irina Margarita Feria-Marrugo¹, Karin Sofía Zúñiga-López²
}

\begin{abstract}
RESUMEN
Esta investigación se enfoca en los objetos virtuales de aprendizaje como herramienta TIC para la enseñanza y el aprendizaje autónomo. Se buscó establecer la relevancia, o no, de ciertas herramientas digitales, representadas como OVA, que cumplieran con ciertas características, para el apoyo en las clases, de tal manera que desempeñaran un papel didáctico para los procesos de enseñanza aprendizaje, el cual demanda metodologías y conocimiento por parte del docente y una actitud deseada por parte del estudiante. Para lograr eso, se planteó como objetivo general de la investigación: "Evaluar la incidencia de los Objetos Virtuales de Aprendizaje como herramienta didáctica digital en la enseñanza y en el desarrollo de aprendizaje autónomo del inglés en los estudiantes de noveno grado de básica secundaria de la Institución Educativa Bertha Gedeón de Báladi". La metodología utilizada fue planteada bajo el enfoque cualitativo y cuantitativo con carácter descriptivo y evaluativo. En la investigación participaron 54 estudiantes de noveno grado de secundaria; los instrumentos utilizados para la recolección de datos fueron encuestas, entrevistas y observación directa, cuya validez fue garantizada implementando un cuadro de triple entrada en el que se establecieron las categorías, los indicadores, los descriptores y los instrumentos de recolección de datos apropiados a la metodología de estudio. Para el análisis de la información se utilizó la triangulación metodológica que permitió tener una visión más compleja de la realidad, por tanto, más completa y cercana a la misma. Los resultados obtenidos revelaron la gran incidencia de los OVA como herramienta motivadora en el aprendizaje autónomo del estudiante y su preferencia ante materiales tradicionales planos, carentes de interactividad o de elementos multimedia que privilegien diversos estilos de aprendizaje.
\end{abstract}

Palabras clave: Objetos virtuales de aprendizaje (OVA), TIC, didáctica, enseñanza, aprendizaje, autonomía.

\section{ABSTRACT}

This research focuses on two main aspects: the virtual learning objects as ICT tool for teaching and independent learning; with this we pursue to establish the relevance or not, of certain digital tools, represented as L0, that had certain characteristics, for support in the classroom, so that they fulfill an educational role for the teaching and learning processes, which demands

Fecha de recepción: Julio 31 de 2015 / Fecha de aceptación: Julio 19 de 2016

Tipología: Artículo de Investigación Científica y Tecnológica

Para citar éste artículo: Feria, M. I. \& Zúñiga, L. K. (2016). Objetos virtuales de aprendizaje y el desarrollo de aprendizaje autónomo en el área de inglés. Praxis. Vol. 12, 63 - 77

1. Magister en Gestión de la Tecnología Educativa. Docente ocasional de tiempo completo, Programa Nacional de Inglés Universidad Nacional Abierta y a Distancia - UNAD, Cartagena de indias, Colombia. Email: irinaferia@gmail.com

2. Magíster en Tecnología Educativa y Medios Innovadores para la Educación. de profesional de producción de contenidos en el Centro de Investigación y Desarrollo en Tecnología de la Información y las Comunicaciones (CINTEL). Bucaramanga, Colombia.Email: karin_sofy@hotmail.com 
methodologies and knowledge by the teacher and the right attitude by the student. Of course, to achieve that, the overall objective of the research is to "Evaluate the incidence of Virtual Learning Objects as digital didactic tool in teaching and in the development of autonomous learning in English in high school students of the Institution Bertha Gedeón Báladi "which was the chosen population. The methodology used was under the qualitative and quantitative approach with descriptive and evaluative. The research involved 54 students high school; the instruments used for data collection were surveys, interviews and direct observation, the validity of which was guaranteed by implementing a table triple entry into the categories were established, indicators, descriptors and instruments for data collection appropriate to the study methodology. For information analysis methodological triangulation that gave a more complex view of reality, therefore more complete and closer to it was used. The results revealed the high incidence of OVA as a motivating tool in the autonomous learning of the student and their preference to traditional materials, instead of resources lacking interactivity or multimedia.

Keywords: Virtual learning Object (LO), Information and Communication Technologies (ICT), Didactic, teaching, learning, autonomy.

\section{INTRODUCCIÓN}

$\mathrm{L}$ as TIC han facilitado procesos y han abierto espacios para nuevas prácticas en diferentes aspectos de la vida del ser humano, según numerosos estudios y escritos que se han venido haciendo en el tema. Desde su aparición a mediados del siglo pasado, las Tecnologías de la Información y comunicación se han expandido en los diferentes entornos de la vida del ser humano, como el entorno personal (diario, cotidiano), el entorno académico y el entorno profesional, es decir, las TIC se han hecho indispensables en actividades básicas de las personas.

Aunque el uso de las tecnologías se está integrando rápidamente en todos los ámbitos, es necesario que desde la educación se tengan en consideración para la formación de las nuevas generaciones, ya que estas las requieren para hacerse competitivas en el siglo XXI. Como lo llama Marqués, P. (2000) la "alfabetización digital” (Computer literacy), requiere añadir nuevos contenidos al currículo con nuevas competencias tecnológicas y culturales necesarias para todos los ciudadanos.

Así como la enseñanza y el aprendizaje van adquiriendo nuevas perspectivas en el tiempo, debido a los cambios en la sociedad, el maestro y el estudiante también han adquirido nuevos roles que les permitan suplir las necesidades y enfrentar los retos que el mundo actual plantea a la educación: uso de nuevas tecnologías, interculturalidad, variados ambientes de aprendizaje, flexibilización, dominio de una lengua extranjera, autonomía en el aprendizaje, por sólo mencionar algunos.

Lo anterior conlleva a una reestructuración de las prácticas pedagógicas, ya que el aula y la biblioteca de la institución educativa, dejaron de ser los únicos espacios donde se encuentra la fuente del conocimiento e información. Con todas las herramientas con las que se cuenta actualmente (canales de TV, ciberbibliotecas e Internet en general), los aprendizajes se dan en cualquier sitio y en la mayoría de los ámbitos que "toca", pero, sobre todo, que llaman la atención del estudiante, ya que lo encuentran más atractivo.

Es por esto que los objetos virtuales de aprendizaje, afloran en este estudio, como recursos digitales, para apoyar los procesos de enseñanzaaprendizaje en el área de inglés de la Institución Educativa Bertha Gedeón de Báladi, dando la posibilidad de tener herramientas adicionales para apoyar la enseñanza y el aprendizaje.

Esta investigación se enfoca en la utilización de Objetos Virtuales de Aprendizaje como una herramienta que permita mejorar los procesos educativos, dentro de ellos, la motivación y el desarrollo de autonomía. Un ámbito educativo mediado por las TIC no es el futuro, es el presente, por lo tanto, como es de esperarse, la educación y sus actores están en una etapa 
de transición donde en muchos casos se está teniendo un beneficio de las tecnologías como apoyo a los procesos, pero en otros, aún no se han contextualizado y se continúa en la actitud de rechazo o indiferencia. En cuanto a la perspectiva metodológica, esta investigación combinó los enfoques cualitativo y cuantitativo, por cuanto describe e interpreta los fenómenos estudiados como procesos propios de la mente y el comportamiento humanos, pero se apoyó en cifras para determinar en qué medida (frecuencia, grado de intensidad, etc.) tales fenómenos están presentes en los sujetos objeto de estudio. Los hallazgos del análisis de los resultados constituyeron un diagnóstico que ratifica el desafío tanto de maestros como estudiantes de enseñar y aprender teniendo en cuenta las habilidades del siglo XXI (Microsoft, 2014).

Teniendo en cuenta lo anteriormente descrito, la presente investigación estuvo guiada por los siguientes interrogantes:

¿De qué manera los Objetos Virtuales de Aprendizaje contribuyen en la gestión académica como herramienta didáctica digital para la enseñanza dentro y fuera del aula de clases?

\section{¿En qué medida la tecnología digital y el contenido multimedia, las herramientas TIC enfocadas a la educación, permiten un espacio de análisis y reflexión a los estudiantes para el desarrollo de aprendizaje autónomo?}

Para resolverlos, surgió la necesidad de analizar las prácticas dadas en esta institución en particular, Bertha Gedeón de Báladi, donde los estudiantes requieren de más recursos educativos para su aprendizaje, ya que en el área de inglés no se cuenta con un texto guía, por lo que sería interesante iniciar un proceso de mediación con los equipos tecnológicos existentes en la Institución, en este caso, cuarenta computadores portátiles y una sala de sistemas con acceso a Internet, donde semanalmente se podrían generar espacios de aprendizaje, tanto para estudiantes como docentes, con el uso de Objetos Virtuales de Aprendizaje que refuercen lo trabajado en el aula, y en otros casos, profundicen e inciten a nuevos conocimientos y mejor comprensión de la lengua inglesa.

\section{Revisión de la literatura}

El estudio partió de la revisión de la literatura existente sobre el tema y la definición de los principales conceptos, así:

Herramientas TIC. Son aquellas herramientas a través de las cuales se busca, procesa, transmite, y transforma todo tipo de información, utilizando en la mayoría de los casos redes como la Internet. La ley 1341 de 2009 define TIC como "el conjunto de recursos, herramientas, equipos, programas informáticos, aplicaciones, redes y medios, que permiten la compilación, procesamiento, almacenamiento, transmisión de información como: voz, datos, texto, vídeo e imágenes”. Las TIC abarcan toda la tecnología que se utiliza para la transmisión y procesamiento de la información, en cualquier campo y para cualquier uso.

\section{Según Cabero (2006):}

Las TIC configuran nuevos entornos y escenarios para la formación con unas características significativas. Por ejemplo: amplían la oferta informativa y posibilidades para la orientación y tutorización, eliminan barreras espacio-temporales, facilitan el trabajo colaborativo y el autoaprendizaje, y potencian la interactividad y la flexibilidad en el aprendizaje. (p. 17 -18)

E-Learning. En español “Aprendizaje electrónico” es un término aplicado a la educación a distancia completamente virtualizada, la cual se basa en la tecnología multimedia con diferentes herramientas de la web 2.0 para la presentación y envío de contenidos. Como se mencionó anteriormente, existen sitios que permiten la creación de espacios virtuales, llamados plataformas, donde los docentes y estudiantes tienen la oportunidad de interactuar. Area \& Adell (2009) afirman que:

Lo característico del e-learning es que el proceso formativo tiene lugar totalmente o en parte a través de una especie de aula o entorno virtual en el cual tiene lugar la interacción profesor-alumnos así como las actividades de los estudiantes con los materiales de aprendizaje. (p.393 - 394). 


\section{Los Objetos Virtuales de Aprendizaje (OVA)}

OA (Objeto de Aprendizaje), LO (Learning Objects) en inglés, los OVA (Objetos Virtuales de Aprendizaje) se convierten entonces en las nuevas herramientas del aula, apoyados, por supuesto, por el conocimiento de las TIC para su implementación.

¿Qué es un OVA? Un Objeto Virtual de Aprendizaje es una unidad de contenido o temática, un material digital para fines específicos de aprendizaje con objetivos, actividades y evaluación. Wiley (2000) caracteriza a los OA como "cualquier recurso digital que pueda ser reusado como soporte para el aprendizaje” (p.6).

Los OVA son herramientas pedagógicas mediadoras de conocimiento, los cuales permiten una presentación didáctica de los contenidos, teniendo en cuenta distintas formas audiovisuales e interactivas. En otro concepto, los Objetos de aprendizaje, son

Entidades digitalizadas encaminadas a lograr el aprendizaje de una competencia, que se configuran didácticamente con objetivos, metodología, contenidos, evaluación, con recursos abiertos (con materiales abiertos que se encuentran con los términos legales para ser usados libremente, con el permiso legal de sus (autores) que se sustentan en las propiedades de reusabilidad, subjetividad, historicidad, comunicabilidad, integralidad, y que se encuentran registrados para el dominio público, liberados bajo un esquema de licenciamiento que protege la propiedad intelectual y permite su libre uso y reúso para la enseñanza, el aprendizaje y la investigación. Ramírez y Valenzuela (2010, p.4)

Un OVA puede ser creado en diversos formatos: HTML, XML, JPEG, PDF, Flash, GIF, WMV, etc., y debería contar con ciertas características que posibiliten su distinción dentro otros recursos didácticos.

Dentro de las características básicas de que deben tener los Objetos Virtuales de Aprendizaje, se tienen las siguientes:
Fiables: Información confiable, verdadera y oportuna según la temática, respetando los derechos de autor.

Interactivos: Responden a diferentes demandas por parte del usuario de forma bidireccional en muchos casos, donde más de un camino es posible para el aprendizaje o utilización de la información.

Reutilizables o reusables: Permiten crear un nuevo OVA a partir de él, ya sea para mejorar su contenido o para utilizarlo en otros contextos.

Compatibles o interoperables: Compatibilidad con otras especificaciones o estándares que permitan su utilización sin inconvenientes técnicos.

Estructurados: Fáciles de utilizar y claros en su presentación (interfaz) para la navegación o exploración por parte del usuario. Una forma práctica es la utilización de hipertexto.

Multimedia: Combinan o se componen de varios medios como imagen, sonido o la suma de ambos (video) para presentar la información.

Otras características importantes que se deben tener en cuenta son:

Atemporales: Para que no pierdan vigencia en el tiempo y en los contextos utilizados, es decir, que pueda actualizarse fácilmente.

Didácticos: El objeto tácitamente responde a qué, para qué, con qué y quién aprende.

Auténticos: Presentan los contenidos de manera diferente, innovadora.

Pertinentes: Pedagógicamente enfocados en las necesidades de los usuarios finales, con unidad de aprendizaje.

Con Diseño: Además de presentar información, lo hacen de forma atractiva utilizando colores adecuados (psicología del color).

En Colombia, el portal educativo Colombia Aprende es un espacio donde se publican 
contenidos educativos digitales, donde estudiantes y docentes interactúan y comparten sus experiencias, proyectos e investigaciones. Todo esto avalado por el Ministerio de Educación Nacional.

\section{Enseñanza y aprendizaje}

Los procesos de enseñanza y aprendizaje van acompañados de diferentes elementos, donde intervienen dos actores principales como lo son el maestro y el estudiante, al igual que otros no menos importantes que enriquecen y caracterizan las acciones del proceso, en esta investigación se mencionan las siguientes:

Enseñanza. Según el diccionario de la Real Academia, la enseñanza es el acto de enseñar, lo que implica dar instrucción, adiestrar, ilustrar, preparar; Es una acción realizada por una persona para otro $u$ otros. La enseñanza hace parte fundamental del proceso de educativo y recae sobre el maestro, ya que, dentro de su rol, enseñar es su labor principal valiéndose de la pedagogía y la didáctica, entre otras, para exponer el conocimiento.

Aprendizaje. Se constituye en la acción de aprender, la cual, contrario a la enseñanza, es un proceso mental, personal e individual, que recae no en el maestro, sino en el estudiante; el aprendizaje es un proceso y un resultado en sí mismo, según Rojas (2001) "es el cambio potencial en una conducta -bien a nivel intelectual o psicomotor- que se manifiesta cuando estímulos externos incorporan nuevos conocimientos, estimulan el desarrollo de habilidades y destrezas o producen cambios provenientes de nuevas experiencias” (p.2).

Didáctica. Se define como "el arte de enseñar", del griego Didaktiké; es una disciplina que se enfoca en el estudio pedagógico de recursos, procedimientos y técnicas de enseñanza para orientar el aprendizaje, es decir, cómo se enseña. Se le atribuye el término a Juan Amós Comenio, el cual es considerado el padre de la didáctica, con su obra Didáctica Magna.

El maestro. Es quien comparte, orienta, promueve, guía, motiva, escucha, ama y aparte de todo tiene la responsabilidad de conocer y entender a sus estudiantes con el fin de optimizar sus procesos de aprendizaje. Es la persona que enseña una ciencia, un arte u oficio.

En el transcurso del tiempo, el maestro ha tenido diferentes denominaciones, entre ellas: maestro, profesor, docente, instructor y tutor.

El estudiante. Es la persona que aprehende, que se motiva para aprender que desarrolla sus capacidades cognitivas en pro de un conocimiento específico; posee diversas inteligencias dependiendo de su especificidad como ser humano y puede apropiarse de diferentes estilos de aprendizaje para lograr sus objetivos de formación. Ha pasado de ser concebido una tabula rasa (tradicionalismo), una copia del maestro (conductismo), un ser que necesita afecto (pedagogías activas), un ser que aprende (teorías cognitivas), un ser que convive (teorías socio constructivistas) a un ser que toma posición y piensa (teorías críticas). Al igual que el maestro, tiene diferentes denominaciones se reconocen las palabras discípulo, estudiante, discente, aprendiz y alumno.

En esta investigación son importantes los cuatro elementos, destacando el entorno, ya que se promueve el E-learning y aprendizaje autónomo, a través de los OVA como herramientas TIC.

\section{Aprendizaje autónomo}

Concepto de autonomía. Como lo indica la palabra autonomía, es la capacidad de ser consciente, independiente, recursivo en la gestión del propio aprendizaje. Una persona autónoma está en la capacidad de dirigir, controlar, regular y evaluar su forma de aprender, esto requiere ser consciente de su estilo de aprendizaje para aprender más y mejor.

La definición clásica de autonomía es la planteada por Holec (1991), quien la describe como "la capacité e l'apprenant de prendre en charge son propre apprentissage” (p. 3), es decir, la capacidad del aprendiente de responsabilizarse de su propio aprendizaje. 
Por otro lado, André (1989), agrega que:

(...) prendre en charge son apprentissage, c'est avoir la responsabilité, et l'assumer, de toutes les décisions concernant tous les aspects de cet apprentissage, c'est-à-dire: la détermination des objectifs; la définition des contenus et des progressions; la sélection des méthodes et des techniques à mettre en œuvre; le contrôle du déroulement de l'acquisition proprement dite (rythme, moment, lieu); l'évaluation de l'acquisition réalisée. L’apprenant autonome est capable de prendre lui-même toutes ces décisions concernant l'apprentissage dans lequel il désire, ou se trouve, être impliqué. (p.3)

La traducción de este extracto comenta que "apoyar su aprendizaje es tener la responsabilidad, y asumir todas las decisiones con respecto a todos los aspectos de este aprendizaje, es decir, la determinación de objetivos; la definición de los contenidos y las progresiones; la selección de métodos y técnicas para poner en práctica; seguimiento de los avances de la adquisición real (ritmo, tiempo, lugar); la evaluación de la adquisición completado. El estudiante independiente es capaz de tomar en sí todas estas decisiones sobre el aprendizaje en el que desea, o es, estar involucrado".

Ser autónomo entonces, implica que hacerse cargo de su aprendizaje es asumir la responsabilidad y las decisiones relacionadas con el proceso como con los objetivos, los contenidos y su progresión; la selección de métodos y técnicas a usar; el control del desarrollo de la adquisición y su evaluación. "El aprendiente autónomo debe ser capaz de tomar por sí mismo todas estas decisiones relativas al aprendizaje que desea adquirir” (Cabrales, Cáseres \& Feria, 2009, p.29).

Objetos Virtuales de Aprendizaje y su incidencia en el proceso de autonomía del estudiante. Dado que el término autonomía es un sustantivo, haría entonces alusión a un estado, una cualidad o una capacidad ya adquirida. Para André (1992), esto significa que: "On n'est pas autonome au départ, mais il y a un processus qui permet sans doute, de le devenir" (p.67). Lo que implica que no es autónomo al inicio, pero hay un proceso que sin duda permite llegar a serlo; es decir, que la autonomización es la práctica de la autonomía.

"Autonomizar" al estudiante es realmente una tarea difícil y uno de los primeros obstáculos es, paradójicamente, el mismo estudiante. Como lo señala Müler-Verweyen (1999):

el estudiante espera siempre que el profesor además de ser competente, experto y generador de motivación, diseñe y controle todas las actividades individuales y del grupo, que formule las tareas y estrategias y las supervise durante su ejecución y finalmente que examine si les metas fueron alcanzadas. (p.79-80)

Según este autor, desde el punto de vista de la autonomía, esta visión del proceso de enseñanza y aprendizaje se constituye en una barrera. ¿Cómo autonomizar al estudiante si las funciones de liderar el aprendizaje no recaen en él y son asumidas por el profesor? La tarea consiste entonces en transferir estas funciones paso a paso para que el estudiante asuma la responsabilidad de su aprendizaje de manera consciente.

Una de las formas de promover esta autonomización, es la implementación de mediadores pedagógicos como lo son los OVA, los cuales permitirían el diseño de unidades de contenidos que podrían adaptarse a los diferentes estilos de aprendizaje de los estudiantes y así empezar a generar cierta motivación, que aparte de promover un aprendizaje significativo, promueva también el desarrollo de autonomía.

De acuerdo con los variados métodos de enseñanza que diferentes autores han propuesto a través de los años, hay algunos como el método tradicional (gramática y traducción para el caso del aprendizaje de una lengua extranjera), que trunca este proceso del desarrollo del aprendizaje autónomo mediado por OVA, ya que el rol del estudiante es pasivo y el docente por su parte, basa el proceso de enseñanza-aprendizaje en la memorización de palabras, lo que reduciría la capacidad cognitiva de los estudiantes y las bondades de la tecnología; a algo que no necesita un medio distinto al tradicional para llevarse a 
cabo. No quiere decir esto que un OVA no puede diseñarse basado en diferentes métodos o enfoques de enseñanza, solo que queda en manos del docente creador o el experto en la temática, guiar las características del mismo, teniendo en cuenta los objetivos a alcanzar. Es por esto tan importante tener en cuenta que el uso de la tecnología debería estar enfocada a la innovación, al desarrollo del pensamiento crítico, a la flexibilidad pedagógica y a la autonomización del estudiante, entre otras. Hay que destacar que de esta temática podría hacerse una investigación completa, donde se estudie y ejemplifique a profundidad, las ventajas y desventajas de utilizar un método obsoleto o actual para la creación de OVA.

\section{METODOLOGÍA}

La investigación se enmarcó dentro de la perspectiva cuantitativa y cualitativa, teniendo en cuenta que se estudiaron y evaluaron los resultados de los procesos en el aula de clase. El enfoque cuantitativo permitió de forma objetiva, analizar a través de encuestas y formatos creados para las actividades modelo para la investigación, es decir, desde una realidad objetiva, concebida desde lo externo. Por otra parte, el enfoque cualitativo se empleó en el sentido de que todo el proceso tuvo una mirada subjetiva, una interpretación, desde un punto de vista interno, desde la observación y la heterogeneidad del grupo. Así mismo, esta investigación es descriptiva-evaluativa, porque buscó describir las prácticas de enseñanza aprendizaje y la relación con el desarrollo de procesos de autonomía acompañado del uso de TIC, Objetos Virtuales de Aprendizaje, además de medir la importancia de estas herramientas tecnológicas como mediadoras pedagógicas en el proceso de enseñanza-aprendizaje del inglés. Para efectos de recolección de información, se utilizaron tres instrumentos: la encuesta, la entrevista y la observación.

\section{Variables}

Conforme se establece en la descripción del problema, esta investigación se enfocó en dos grandes aspectos: Objetos Virtuales de Aprendizaje para la enseñanza y el desarrollo del aprendizaje autónomo. Para cada categoría se determinaron indicadores que constituyen las características identificadoras de los procesos y elementos en estudio. Son esos indicadores los que facilitan la descripción detallada de los mismos para así poder determinar en qué medida los estudiantes son o no autónomos y por qué razones el uso de las TIC en la enseñanza se constituye en un factor clave en la inducción a la autonomía.

\section{Tabla 1.}

Operacionalización de las variables

\begin{tabular}{|c|c|c|c|}
\hline Variables & Categorías & Indicadores & $\begin{array}{c}\text { Técnicas e } \\
\text { Instrumentos }\end{array}$ \\
\hline $\begin{array}{l}\text { Objetos virtuales } \\
\text { de Aprendizaje }\end{array}$ & $\begin{array}{l}\text { Pertinencia } \\
\text { Unidad de } \\
\text { aprendizaje }\end{array}$ & $\begin{array}{l}\text { Utilización de herramientas y } \\
\text { aplicaciones desde Internet para } \\
\text { apropiación y distribución del } \\
\text { conocimiento. }\end{array}$ & $\begin{array}{c}\text { Bitácora de } \\
\text { herramientas OVA } \\
\text { Blog de inglés }\end{array}$ \\
\hline $\begin{array}{l}\text { Desarrollo de } \\
\text { aprendizaje } \\
\text { autónomo }\end{array}$ & $\begin{array}{l}\text { Gestión del } \\
\text { aprendizaje }\end{array}$ & $\begin{array}{c}\text { Planificar las tareas, auto-gestionar } \\
\text { el aprendizaje, auto-monitorear el } \\
\text { desarrollo de trabajos. } \\
\text { Ampliar y profundizar sus } \\
\text { conocimientos sobre temas tratados. }\end{array}$ & $\begin{array}{l}\text { Encuesta a } \\
\text { estudiantes. } \\
\text { Resultados de } \\
\text { trabajos. }\end{array}$ \\
\hline
\end{tabular}




\section{Continuación Tabla 1.}

\begin{tabular}{|c|c|c|c|}
\hline Variables & Categorías & Indicadores & $\begin{array}{c}\text { Técnicas e } \\
\text { Instrumentos }\end{array}$ \\
\hline $\begin{array}{l}\text { Uso de las de } \\
\text { las herramientas } \\
\text { TIC en el proceso } \\
\text { de enseñanza } \\
\text { aprendizaje. }\end{array}$ & $\begin{array}{l}\text { Conocimiento o } \\
\text { capacitación en } \\
\text { manejo de TIC. }\end{array}$ & $\begin{array}{c}\text { Integración de proyectos y programas } \\
\text { con las TIC. } \\
\text { Familiarización y actitud del } \\
\text { alumnado y sus familias hacia las } \\
\text { tecnologías. } \\
\text { Actitud del profesorado ante la } \\
\text { tecnología. }\end{array}$ & $\begin{array}{c}\text { Guía de entrevista } \\
\text { a docentes de la } \\
\text { Institución. }\end{array}$ \\
\hline
\end{tabular}

Fuente: Elaboración propia

\section{Población y muestra}

La población objeto de estudio de esta investigación, estuvo constituida por estudiantes de noveno grado de secundaria de la Institución Educativa Bertha Gedeón de Báladi. Asimismo, se definió una muestra aleatoria de 54 estudiantes. Con esto, se buscó tener una visión completa de todos los estudiantes de noveno grado de secundaria de la Institución, en relación con el uso de las TIC y su relación con el desarrollo de la autonomía. Estos jóvenes pertenecen en su mayoría a los estratos 1 y 2 y tienen edades que oscilan entre los 14 a los 17 años.

\section{Procedimiento}

Esta investigación se estructuró en tres fases a fin de lograr el cumplimiento del objetivo general "Evaluar la incidencia de los Objetos Virtuales de Aprendizaje como herramienta didáctica digital en la enseñanza y en el desarrollo de aprendizaje autónomo de inglés en los estudiantes de noveno grado de básica secundaria de la Institución Educativa Bertha Gedeón de Báladi”. En este sentido, en la primera fase se llevó a cabo la documentación, indagación y exploración acerca de la relevancia de los Objetos Virtuales de Aprendizaje en la enseñanza en básica secundaria, la segunda fase consistió en llevar a la práctica la utilización de OVA en la clase de inglés, donde la plataforma a utilizar como soporte de los OVA fue el blog http://inglesberthagedeon.blogspot.com; por último, la tercera fase correspondió a la evaluación, en la cual se valoró la incidencia del uso de TIC en el proceso.

\section{RESULTADOS}

El análisis de los datos obtenidos, se estructuró en tres grandes partes: Dos correspondientes a las variables que fueron objeto de estudio: la utilización de Objetos Virtuales de Aprendizaje en la enseñanza y aprendizaje y la autonomía de los estudiantes en el aprendizaje del inglés, y uno relativo a la utilización de herramientas TIC por parte de docentes a través de entrevistas aleatorias a docentes de la institución. El análisis se hizo sobre datos obtenidos por instrumentos:

- Encuesta dirigida a estudiantes, conformada por 9 preguntas de selección con única respuesta, cuyo objetivo fue recopilar información que permitiera conocer la opinión de los estudiantes de básica secundaria de la Institución Educativa Bertha Gedeón de Báladi, en cuanto al proceso de aprendizaje del inglés con relación al uso de Objetos virtuales de Aprendizaje y su influencia en el desarrollo de procesos de autonomía.

- Entrevista dirigida a docentes, conformada por 6 preguntas abiertas, enfocada en recopilar información que permitiera determinar qué actitud tienen los docentes con relación a las TIC en la educación, en qué medida las consideran importantes el uso para su asignatura y qué tan familiarizado están con ellas. Además, indagar acerca de lo que docentes consideran que aporta el uso de TIC para el desarrollo de la autonomía de los estudiantes.

- Guía de observación de clases dirigida a docentes, que incluye 3 variables que permitieron observar el comportamiento de los 
estudiantes en clases apoyadas con TIC e identificar su percepción y motivación en cuanto al uso de ellas.

\section{Objetos virtuales de aprendizaje y desarrollo de procesos de autonomía (Encuesta dirigida a estudiantes)}

\section{La Internet y algunas aplicaciones digitales para uso educativo}

Se sabe que la Internet y específicamente los buscadores web son una excelente herramienta para la búsqueda de información de todo tipo, pero cuando se quiere enfocar esas búsquedas a lo académico, ¿qué tan fácil es encontrar información para los estudiantes?

Algo menos de la mitad del curso afirma que la Internet y aplicaciones le ayudan "Mucho" en su gestión académica, es decir, los estudiantes utilizan Internet y aplicaciones como una forma de refuerzo para mejorar académicamente. Si a esta proporción se agrega el $37 \%$ de aquellos que contestaron "Medianamente", se tiene un 79,6\% de los estudiantes que indican que regularmente se acude a la Internet con fines educativos, lo cual es satisfactorio.

Sigue en proporción la frecuencia "Poco" con el $13 \%$, es decir, sólo en algunos casos los estudiantes acuden a Internet y aplicaciones con el fin de mejorar académicamente, sumándose el 7,4\% corresponde a la opción "Muy poco", dada por lo estudiantes que tal vez no tienen los medios, no saben cómo utilizar internet para la investigación académica, al igual que los dos últimos casos, donde podría interpretarse que estos efectivamente no cuentan con los medios para el acceso a Internet o equipos.

\section{Materiales Educativos online}

Muchas veces, este gran flujo de datos que se encuentra en la red desinforma y confunde, por lo que esta segunda pregunta, indagó acerca de lo útil que encuentran la información online.

Según los resultados de la encuesta, sólo la mitad de los estudiantes, equivalente al $50 \%$, encuentran los materiales, explicaciones, recursos u otra herramienta en línea que faciliten el entendimiento de ciertos tópicos. Esto puede interpretarse de dos formas: o no hay suficiente material educativo con metadatos que permitan su fácil búsqueda, o los estudiantes no saben buscar los contenidos educativos de una forma efectiva. En este caso, lo ideal es que esta proporción corresponda a aquellos que sí encuentran los materiales con éxitos, pero el porcentaje equivalente a este ítem es 33,4\% .

Las otras opciones que fueron escogidas, pero esta vez en menos proporción fueron "Rara vez" con un $9,2 \%$ y "No" con $7,4 \%$, lo que se traduce en cinco y cuatro estudiantes de la muestra respetivamente; lo cual puede tratarse de estudiantes que no tienen un acceso diario a la web y por tanto, no hace búsqueda de información online.

Estudio independiente con ayuda de herramientas digitales

Más de la mitad del grupo manifiesta que es una ventaja contar con la ayuda de herramientas digitales en casa, eso representa el 68,5\% de los estudiantes. Teniendo en cuenta lo atractivo que encuentran muchas veces los estudiantes un ambiente virtual, es un alto porcentaje el que considera que no es una ventaja apoyarse en herramientas digitales. Al tratarse de una respuesta semiabierta, muchos respondieron el por qué y estas fueron las coincidencias que se encontraron en ellas:

Estudiantes que responden “Sí, argumentan que, al utilizar la Internet en casa tienen acceso a más información de la que fue recibida en el aula de clases, del mismo modo es información más actualizada que la que encuentran en los libros; también se resalta que hay más opciones (variedad) para contrastar un mismo tema y se encuentra más rápido y fácil, esto puede deberse a los filtros que ofrecen los buscadores, y además se profundiza en el conocimiento, dando como resultado un mejor aprendizaje.

Por otro lado, los estudiantes que respondieron "No", un grupo conformado por el 31,5\%, afirman que los libros siempre serán mejores ya que 
se ven obligados a leer y no a copiar, no tienen la tentación de distraerse con otras páginas, publicitad, chats, correo, etc., y que además, la información de los libros es más confiable, lo que puede interpretarse como que en Internet puede que no se publiquen investigaciones, sino supuestos de quienes escriben.

\section{Utilización de blog personalizado para estudio y consultas}

En la clase de inglés se creó un blog para consulta. Regularmente los estudiantes acudían al blog a hacer repaso, mirar videos, socializar en el chat, o estudiar algún tema de la clase, por lo que, al ser una herramienta de la web 2.0, se quiso saber la relevancia del mismo en su proceso de aprendizaje.

El mayor porcentaje corresponde a la respuesta "Sí", lo que evidencia que el 94,4\% de los estudiantes encuentran beneficioso contar con una herramienta que les proporciona ayuda fuera del aula. Al argumentar el porqué de su respuesta, aquellos que escogieron "Sí" afirman que pueden estudiar en casa y recodar lo visto en clase; comprenden mejor los temas, es más didáctico, encuentran lo que necesitan.

En oposición, el 5,6\%, tres estudiantes, sostienen que les va mejor con explicaciones personalizadas, por lo que la respuesta "No", es la apropiada según su estilo de aprendizaje.

\section{Recursos pedagógicamente creados que más contribuyen al estilo de aprendizaje}

Según los resultados de la encuesta, los recursos más provechosos por los estudiantes del IE Bertha Gedeón de Báladi son tres: Videos, juegos y actividades interactivas, que en porcentajes se darían en un $23,3 \%, 23,3 \%$ y $19,4 \%$ respetivamente. Estos tres recursos, podría decirse, no son comunes en la enseñanza tradicional, pero da cuenta de que sí lo son para los estudiantes actualmente.

En más baja proporción, prefieren imágenes con texto, presentaciones (power point, slide share), audios o libros multimedia, con porcentajes de $10,7 \%, 10,7 \% 8,7 \%$ y $3,9 \%$ respectivamente. Puede ser coincidencia o no, pero son precisamente los recursos que son planos, que no hay movimientos o interactividad.

En la opción de respuesta “otra”, dos estudiantes correspondientes al 3,7\% de la muestra, coincidió en que otra de las formas con que suelen aprender es a través de la música, lo que es algo bastante importante, ya que es una forma natural de acercarse a la lengua inglesa, además de la cultura como tal, con artistas, frases, pronunciación, estilo musical, etc.

\section{Ventajas de OVA (Objetos Virtuales de Aprendizaje) con diseño pedagógico en el área de inglés}

Luego de constatar la preferencia de los estudiantes con relación a videos, juegos y actividades interactivas, se les pregunta qué ventajas encuentran en los recursos escogidos, ya que según el estilo de aprendizaje de cada cual, la estrategia que representa cada uno puede ser diferente y los procesos se dan de una mejor manera.

El 25,5\% de los estudiantes consideran que los recursos educativos que contienen imágenes y/o sonidos, le facilitan su aprendizaje, es decir, son conscientes de que, a través de ellos, los procesos formativos se dan de una mejor manera, siendo ésta la respuesta con mayor porcentaje de aceptación en cuanto a ventajas de utilizar OVA en el área de inglés se refiere. El aprendizaje de una lengua extranjera requiere en gran parte de una buena memoria o en su defecto, buenas estrategias que ayuden a la retención de la información. Ésta es la razón número dos por la cual los estudiantes aprenden más rápido con ciertas estrategias, porque les ayuda a memorizar, por ejemplo. Un $22,6 \%$ así lo afirman, ya que consideran que se divierten y aprenden a la vez. La lúdica es una metodología exitosa, en cuanto los estudiantes no sienten obligación, sino al contrario, un placer al aprender. Esto coincide con el hecho de que las estrategias que emplean no son las más tradicionales, propias de un aprendizaje memorístico. 
Por otro lado, el 18,6\%, afirman que la utilización de ciertas estrategias les ayuda a aprender a su propio ritmo, en este caso, cuando se tienen recursos disponibles fuera del aula, que pueden ser reutilizados, estudiados, los estudiantes no sienten la presión de tener que ir al ritmo de la clase, sino que tienen la posibilidad de releer y analizar, escuchar, ver, hasta estar seguros que han comprendido.

Otros estudiantes sostienen que visualizan los temas de diferente forma, este grupo corresponde al $12,7 \%$ y la opción "Puedo releer y repetir la explicación varias veces”, que está relacionada con la anterior, son también ventajas de los contenidos virtuales, ya que se encuentran en un escenario diferente a la clase, son objetivos y responden a un núcleo temático específico que ayuda al estudiante con su aprendizaje.

Tan sólo un $8 \%$ sostiene que aumenta su interés en la clase. Este aspecto de la motivación es muy importante para los procesos de autonomía, por lo que habría que revisar el tipo de información y estructura de los recursos virtuales, de manera tal que los estudiantes sientan curiosidad y motivación por interactuar con ellos y por ende, sacar un mejor provecho del estudio independiente.

\section{Gestión de aprendizaje}

Este aspecto está estrechamente relacionado con la autonomía del estudiante, en la medida en que es un proceso individual y consciente en cuanto a la organización del tiempo, las acciones guiadas para llevar a cabo su aprendizaje, las consultas más allá de lo estrictamente requerido en clase, auto evaluarse, cooperar con otros; son algunas de las que un estudiante con cierto grado de autonomía está dispuesto hacer y que, de hecho, lo hace sin ser consciente de ello. El cuadro de este ítem muestra los siguientes resultados:

Se concluye entonces que sólo el 14,8\% de los estudiantes estaría motivado en profundizar fuera de la Institución Educativa, lo que supone una baja proporción si se compara con el 55.6\% que escogió la opción “Más o menos”, lo que se traduce en una acción no constante y que sólo se realiza en algunos casos. En menor frecuencia, el 7,4\% y 22,2\%, corresponden a la selección "Рoco" y "Muy poco", que suman el total de 16 estudiantes de la muestra.

Para el caso del desarrollo de autonomía, el porcentaje es aún bajo, ya que aproximadamente el $85 \%$ de los estudiantes no están haciendo suficiente fuera del aula, para mejorar académicamente, consultar, y/o tal vez, no cuentan con una orientación clara y motivante para hacerlo. Está la situación hipotética de que, en cada asignatura, contaran con recursos accesible desde cualquier lugar, con ejercicios y presentación de temáticas en formas variadas, itendrían mejor actitud con relación al estudio?

\section{Acciones comunes en la gestión de aprendizaje}

Este ítem corresponde a la forma como los estudiantes materializan la consulta para profundizar sus conocimientos, las respuestas a la pregunta número 8 indican lo siguiente:

Los mayores porcentajes, en relación con el número total de respuestas (74), se ubican en las opciones: consultas en Internet $(41,9 \%)$ y materiales audiovisuales $(29,7 \%)$ con un total de estas dos categorías del 71,6\%, lo cual muestra que la utilización de tecnología es influyente como medio de apoyo para lograr desarrollos en la autonomía del aprendizaje. A pesar de que el chat y correo electrónico exige un alto grado de interactividad, sólo el $4 \%$ tomó esta opción, puede deberse a que se especificó “en inglés”, lo que dicta que a pesar de que lo puedan utilizar para varias actividades académicas, en la lengua inglesa lo hacen con menos frecuencia.

En cuanto a las opciones que exigen mayor reflexión sobre los contenidos por aprender: consultas en biblioteca, grupos de estudio, grupos de investigación, tutorías con docentes, sumados sus porcentajes, estos solo corresponden al $15,9 \%$ del total de las respuestas.

La última opción de la pregunta "No aplico" se vio representada por un $8,2 \%$ de los estudiantes, los cuales con toda sinceridad expresan que no se preocupan en profundizar y/o poner en práctica los cocimientos recibidos en el aula. 


\section{¿Soy un estudiante autónomo? Opinión de estudiantes}

A manera de conclusión de la encuesta, la última pregunta (9) pide a los estudiantes decir en qué medida se consideran autónomos (poco, en gran medida). Los resultados son los siguientes:

En esta gráfica se observa que un $25,9 \%$ de los estudiantes se consideran autónomos y un porcentaje igual estima que lo son en gran medida. Desde la perspectiva de la autonomización en el proceso del aprendizaje de una lengua extranjera, lo ideal sería que el $37 \%$ se considerara muy autónomo y el 25,9\% se calificara como "Рoco" autónomo. Pero lo positivo de estos resultados es que sumando los ítems "Sí” y "En gran medida”, se tiene un $51,8 \%$ de los estudiantes que consideran estar en el camino desarrollo de autonomía.

Podría entonces decirse que se necesita más motivación intrínseca y extrínseca para elevar estos niveles de autonomía, y una de las formas que se propone, es la creación de Objetos Virtuales de Aprendizaje que incentiven y motiven al estudiante a interactuar con el contenido, analizar y profundizar lo estudiado en el aula. Por otro lado, es importante que en este punto, los docentes también sean conscientes del verdadero sentido de la autonomía, y no atribuir manifestaciones erradas.

Uso de las de las herramientas TIC en el proceso de enseñanza aprendizaje (entrevista a docentes)

En general, dentro de los profesores entrevistados, hay una opinión en común y es la importancia de la incursión de las herramientas tecnológicas como mediación pedagógica. Unos expresan que son una oportunidad para reformar las metodologías, otros que son necesarias para ajustarse al ritmo de los estudiantes, también mencionan que es primordial contextualizar la educación. Esto demuestra un interés y a la vez una preocupación por utilizar de la mejor forma los recursos disponibles. En cuanto a las herramientas TIC utilizadas, en algunos docentes no hubo claridad, ya que las confundían con los equipos electrónicos, más específicamente el VideoBeam, pero hubo otros que sí mencionaron algunas como scratch para la clase de inglés, las Webquest en clase de lengua castellana; uno de los docentes expresa que le gustaría incluir el blog para una interacción fuera del aula y una construcción colectiva del conocimiento en clase de química.

Con relación a la motivación, acuerdan que sí la incrementa, ya que los estudiantes encuentran las clases más llamativas e interesantes y les despierta las ganas de investigar, de socializar, de compartir.

Finalmente, las dos últimas preguntas relacionadas con el uso educativo que se le da a las TIC y el desarrollo de autonomía, algunos consideran que no se trata de darles un mal o buen uso de las TIC, sino que dentro de sus conocimientos hacen lo que pueden, pero que la capacitación docente debería ser una prioridad. En cuanto a la creación de OVA en su asignatura, pocos lo han hecho, según expresaron, la mayoría utiliza material terminado que encuentran en la red, pero que les parecía muy interesante iniciar esta labor para tener una especie de repositorio de consulta para sus estudiantes.

Luego de analizar estas respuestas dadas por los docentes, se destaca el hecho de la preocupación de muchos por querer hacer lo que requiere $\mathrm{y}$ demandan sus estudiantes según las necesidades de la sociedad, ya que la mayoría reconoce las herramientas TIC, no como una moda, sino como la herramienta de apoyo para una educación de calidad. Al referirnos a los OVA, muchos preguntaron qué eran y luego de hablar un poco, admitieron que es menester que cada docente pueda aprender a crearlos y contextualizarlos para sus estudiantes.

En general, lo que se despertó también con esta entrevista, fueron dudas y deseos de investigar un poco más el tema para ponerlo en práctica en clases.

\section{Observación de clases}

Se observaron dos clases, en las cuales se utilizaron dos OVA creados con la intensión investigativa, uno para observar los procesos de 
autonomía, el otro para evaluar la pertinencia de un OVA, su contenido y características y que tanto permiten el éxito o no, de un proceso de enseñanza aprendizaje.

En la primera observación, con el tema de gustos y disgustos (Likes and dislikes), fue notoria la respuesta de los estudiantes, cuando sienten que hay una preparación y que los recursos utilizados, responden a sus intereses y expectativas. Este OVA les gustó mucho y ratifica lo importante que es que un OVA cuente con una unidad de aprendizaje, personalización y didáctica, ya que los estudiantes perciben que fue algo hecho pensando en ellos. Con la utilización del segundo OVA, se buscó que los estudiantes tuvieran un soporte para su clase de inglés, que pudieran consultar para profundización y de igual forma delegar una responsabilidad en ellos, para que la clase fuera un escenario de ida y vuelta y sus dudas una guía para la retroalimentación.

Los estudiantes se mostraron motivados y el flujo de la clase se hizo de manera exitosa. Fue un incentivo para los estudiantes aprender de la misma manera como se desenvuelven en sus labores cotidianas en la red; de igual forma, son un apoyo inmenso para el docente, los OVA preparados para las actividades. En ambos casos se evidenció lo que se ha reiterado en esta investigación, y es que un OVA bien realizado, puede moldear no sólo una clase, sino la metodología para ajustarla a las demandas del siglo XXI.

\section{DISCUSIÓN}

Luego del análisis y la interpretación de la información obtenida se procedió a contrastar la literatura con los resultados de esta investigación. Para ello, conviene seguir el orden de las variables propuestas y de igual forma, con los objetivos, lo que le dará una secuencia lógica al proceso.

En primera instancia, los Objetos Virtuales de Aprendizaje se consideran por parte de los estudiantes como una herramienta que les ayuda en gran medida en la comprensión y la consulta y aumentan la motivación en el área o tema. Estas herramientas didácticas, son buscadas en
Internet, pero es claro que aún se tienen falencias en el registro de metadatos por parte de los creadores, que ayuden a encontrar fácilmente los OVA subidos a la nube. Debido a esto, no logran su búsqueda fácil, por lo que, en la encuesta aplicada, respondieron que sólo algunas veces era efectiva la búsqueda y muchas veces se desperdician los recursos ya creados y que pueden resolver en un gran porcentaje las dudas consultadas en la Internet. Por tanto, es necesario un conocimiento básico de búsqueda académica en el ciberespacio, que permita aprovechar al máximo las consultas de diferentes temas a través de presentaciones multimedia en la web.

Por otro lado, los jóvenes encuestados, son considerados usuarios frecuentes de la Internet, y consideran importante la forma como son presentados los contenidos ya que algunos contribuyen a su estilo de aprendizaje. Esto es bastante interesante, porque un OVA puede privilegiar la audición, visión, lectura o cinestesia, lo que para algunos se traduce en ventajas para el aprendizaje del idioma inglés.

En cuanto al desarrollo del aprendizaje autónomo, más de la mitad de los estudiantes comentan que los OVA ayudan en su proceso de aprendizaje y que por los diferentes estilos, unos prefieren algunos recursos por encima de otros, pero que en general son bastante útiles en la gestión de su aprendizaje, aunque sólo en $50 \%$ de ellos se considera realmente autónomo.

Por último, por parte de los docentes se evidencia un gran interés en implementar o mejorar las prácticas en el aula con la ayuda de OVA, muchos de ellos conocen sólo algunas herramientas para hacerlo, pero están en gran disposición de actualizarse y empezar a crear sus propios recursos digitales; en este punto todos coinciden en que la falta de tiempo es un factor que se le suma a la falta de experticia en el tema, al igual que la poca exploración de los recursos ya creados donde pueden constatar la utilidad del uso de las herramientas TIC en el proceso de enseñanza aprendizaje. La educación mediada por la tecnología es una oportunidad para el crecimiento personal y profesional del docente, al igual que un elemento clave para autonomía, la calidad, la 
colaboración, el desarrollo social, la innovación, como lo señalan diversos autores reconocidos en sus investigaciones como Castells, Adell, \& Area (2013), entre otros.

Por lo anterior descrito se denota la complejidad del proceso educativo y su carácter holístico, puesto que todos los factores que intervienen en él están íntimamente relacionados; en este caso: Los OVA, el aprendizaje autónomo y la capacitación de docentes para el correcto uso y creación de herramientas TIC, es por esto que conviene reflexionar al respecto y empezar a tener una mirada crítica con relación al desempeño como profesional, al rendimiento como estudiante y a la pertinencia de los procesos propuestos en el aula, si realmente son significativos y si aportan para el desarrollo de competencias que los estudiantes requieren.

\section{CONCLUSIONES}

A manera de conclusión, se puede entonces reafirmar que en Colombia estamos en una etapa inicial en el proceso de creación y capacitación en el uso de recursos digitales que conlleven a una mejora en los procesos educativos; que en general los estudiantes no se han habituado a ejercer un control sobre la gestión del aprendizaje, sobre los procesos cognitivos, ni sobre los contenidos de estudio, porque no se han creado las condiciones para que ellos desarrollen esas competencias. Que los docentes requieren de más capacitación para la creación del material necesario como complemento de sus clases presenciales y el aprovechamiento de la Web con fines educativos.

Las conclusiones de la presente investigación conducen a formular algunas recomendaciones tendientes a aportar orientaciones para la solución de la problemática tratada en este proyecto, las cuales se expondrán en forma breve en este apartado.

En primer lugar, se hace evidente la necesidad de dar a conocer los resultados de esta investigación tanto a los estudiantes como a los docentes con el fin de que ambos estamentos inicien un proceso de reflexión y búsqueda de formas que los conduzcan a superar las debilidades puestas aquí en evidencia. Pero el compromiso de diseñar e implementar estrategias orientadas a la solución de la situación no debe ser individual, de uno o más grupos, sino colectivo e incluso institucional.

Tal como se expresó en el planteamiento del problema y en la justificación de esta investigación, se requieren más espacios para la investigación y actualización de currículos que hagan estudiantes y profesores más competitivos, para eso es importante que se sigan evaluando las posibilidades de mejora de la infraestructura de la institución (Esto es un limitante), donde los pocos recursos con los que se cuenta, puedan ser utilizados al máximo; no es fácil la creación de una sala de informática, pero para los docentes de todas las áreas, es importante un espacio como ese, para poner en práctica lo que vayan aprendiendo y de esa forma, enriquecer y mejorar junto con sus estudiantes, las experiencias del aula.

Con la satisfacción del deber cumplido, se culmina este proyecto de investigación que espera ser la base de otras investigaciones y proyectos y sobretodo del mejoramiento de la calidad de la enseñanza y el aprendizaje en la Institución Educativa Bertha Gedeón de Báladi.

\section{REFERENCIAS BIBLIOGRÁFICAS}

André, B. (1992). De l'autonomisation à l'autonomie en didactique des langues non maternelles. En: Porcher, L. (Coord.) Le français dans le Monde: les autoapprentissages (pp. 66-74). Paris: Clé International.

Area, M. \& Adell, J. (2009). —eLearning: Enseñar y aprender en espacios virtuales. En J. De Pablos (Coord): Tecnología Educativa. La formación del profesorado en la era de Internet. Aljibe, Málaga: 391-424.

Cabero, J. (2006). Bases pedagógicas para la integración de las TIC en Primaria y Secundaria. Universidad de Sevilla: Grupo de Tecnología Educativa de la (http://tecnologiaedu.us.es/ bibliovir/pdf/ Bases - 456.pdf) (04-09-2009). 
Cabrales, M., Cáseres, J. \& Feria, I. (2009). La autonomía en el aprendizaje del inglés en los estudiantes del ciclo básico del programa de lenguas modernas de la universidad de san buenaventura. Universidad de San Buenaventura Cartagena, Cartagena de Indias, Colombia.

Castell, M., Adell, J., \& Area, M. (2013). Ludoliteracy, creación colectiva y aprendizajes. II Congreso internacional educación mediática y competencia digital. Barcelona. España.

Holec, H. (1991). Autonomie de l'apprenant: de l'enseignement à l'apprentissage. CRAPEL, Université de Nancy 2. Education permmanente No. 107: 3.

Marquez, P. (2000). Competencias básicas y alfabetización digital. Universidad Autónoma de Barcelona. Recuperado de: http://peremarques. pangea.org/competen.htm

Microsoft. (2014). "Habilidades Siglo XXI su impacto en la práctica áulica". Curso "Transformando la práctica educativa docente”. Qualitas Red Tecnologica.
Müler-Verweyen, M. (1999). Reflections as a means of acquiring autonomy. EN: COTTERAL, Sara and CRABBE, David (Ed.). Learner autonomy in language learning: Defining the field and effecting change. Frunkfurt: Peter lang: 79-80.

Ramírez, M. S. \& Valenzuela, J. R. (2010). Objetos de aprendizaje abiertos orientados a desarrollar competencias docentes para la Sociedad del Conocimiento. Ponencia presentada en Edutec 2010 "E---learning 2.0: Enseñar y Aprender en la Sociedad del Conocimiento", Bilbao, España.

Rojas, F. (2001). Enfoques sobre el Aprendizaje. Universidad Simón Bolívar. Recuperado de http://especializacion.una.edu.ve/teoriasaprendizaje/paginas/Lecturas/Unidad \% 202/ rojas2001.pdf

Wiley, D. (2000). Connecting learning objects to instructional design theory: a definition, a metaphor, and a taxonomy. The Instructional Use of LEARNING OBJECTS: Online Version 2000. Recuperado de http://www.reusability. org/read/ 